九州大学学術情報リポジトリ

Kyushu University Institutional Repository

\title{
Local Instability of an Elliptical Flow Subjected to a Coriolis Force
}

Naing, Me Me

Faculty of Mathematics, Kyushu University

Fukumoto, Yasuhide

Faculty of Mathematics, Kyushu University

http://hdl. handle. net/2324/14615

出版情報 : MI Preprint Series. 2009-18, 2009-11-25. The Physical Society of Japan バージョン：

権利関係 : 


\title{
MI Preprint Series
}

Kyushu University

The Grobal COE Program

Math-for-Industry Education \& Research Hub

\section{Local Instability of an Elliptical Flow Subjected to a Coriolis Force}

\author{
Me Me Naing \& Y. Fukumoto
}

MI 2009-18

( Received May 15, 2009 )

Faculty of Mathematics

Kyushu University

Fukuoka, JAPAN 


\title{
Local Instability of an Elliptical Flow Subjected to a Coriolis Force
}

\author{
Me Me Naing and Yasuhide FUKUMOTO
}

Faculty of Mathematics, Kyushu University 33, Fukuoka 812-8581

\begin{abstract}
We make the local stability analysis of a rotating flow with circular or elliptically strained streamlines, whose rotation axis executes constant precessional motion about an axis perpendicular to itself, based on the WKB method. In the frame rotating with the precessional angular velocity, the basic flow is a steady velocity field linear in coordinates in an unbounded domain. For the case of slow precession, without strain, the growth rate takes the same value as that of Kerswell (1993) though the basic flow is different. We find that, in the absence of strain, the growth rate approaches the angular velocity of the basic flow as the precessional angular velocity is increased. The cooperative action of the weak Coriolis force and the elliptical straining field is investigated both numerically and analytically. An analysis of using the Mathieu method reveals that the elliptical instability is weakened by the precession, while the precessional instability is either enhanced or weakened depending on the orientation of the strain.
\end{abstract}

KEYWORDS: local stability, rotating flow, elliptical instability, precession, Coriolis force

\section{Introduction}

The elliptical instability has been established as a universal mechanism for local instability of a rotating flow, with elliptically distorted streamlines, to three-dimensional disturbance of short wavelength. Bayly ${ }^{1}$ noted that the stability problem of a steady velocity field linear in coordinates can be reduced to a system of ordinary differential equations. A general solution of the Euler equations linearized in disturbance amplitude is a linear superposition of the Floquet modes and the growth rate is given by the exponents of a matrix Floquet problem. The numerical analysis of the Floquet problem revealed that the elliptical flow is linearly unstable to three-dimensional disturbances. A through asymptotic analysis was made by Waleffe. ${ }^{2}$ As an extension of Bayly's analysis, the geometric optics (WKB) theory for time-evolution of localized disturbances was developed ${ }^{3,4}$ and now becomes a standard tool for studying the local stability of general flows. A stability criterion is supplied by the solution of the initialvalue problem of a system of the characteristic equations.

The stabilizing and destabilizing effect of the Coriolis force on the elliptical instability, with the axis of the rotating frame being parallel to the vorticity vector of the basic flow relative to it, was comprehensively studied. ${ }^{5-7}$ Understanding of response of rotating flows to precession has received attention from the viewpoints of planetary flows and dynamo. The stability of precessing flows with velocity field linear in coordinates, with streamlines projected 
to the plane normal to the rotation axis being of circular shape, was examined by Kerswell ${ }^{8}$ and Mahalov, ${ }^{9}$ and an instability of the precession origin was found.

The present study first revisits the local stability of a rotating flow, with circular streamlines, whose rotation axis executes a precessing motion about the axis perpendicular to itself. In the rotating frame, the basic flow is realizable as a steadily rotating flow, subjected to the external Coriolis force, supplemented with a simple shear flow along the main rotation axis. This is the same flow field as in ref 9, except for presence of a cylindrical boundary in the latter, for which a global stability analysis was made. This basic flow takes different form from that of ref 8 , but the growth rate is common for the case of small precession angular velocity. Second, we enter into the regime of large precession angular velocity and uncover the new instability mechanism. Third, we look into a combined influence of the precession and elliptical distortion of streamlines.

The detective work on instabilities of our model is threefold. After a brief formulation of the WKB method in $\S 2$, we explore in $\S 3$ the stability of a rotating flow subject to precession in the absence of elliptical deformation of streamlines. We make a separate treat of the cases of slow and fast precession in $\S 3.1$ and $\S 3.2$ respectively. In $\S 3.2$, we manifest a new instability mechanism prevailing a rotating shear flow. In $\S 4$, with circular streamlines, as the second step, we explore the stability of rotating flows subject to fast precession. Finally, $\S 5$ describes the influence of cooperative action of the Coriolis force and the elliptical straining field, both being week, upon stability of a rotating flow.

\section{Formulation and WKB characteristic equations}

Let us consider an inviscid incompressible rotating flow whose velocity field is linear in coordinates, with angular velocity equal to unity, and whose rotating axis itself is rotated about an axis perpendicular to itself. Put it in another way, we are concerned with the stability of a local region in a rotating flow subjected to an external Coriolis force, stemming from precession. The external Coriolis force alters the basic flow with closed streamlines inducing a simple shear flow along the rotating axis of the basic flow. In the rotating frame in which the basic rotating axis is fixed, the velocity and pressure fields $\boldsymbol{u}$ and $p$ satisfy the Euler equations augmented by the Coriolis force. Introduce Cartesian coordinates $(x, y, z)$, in the frame rotating about the $x$-axis with angular velocity $\boldsymbol{\Omega}=(\epsilon, 0,0)$, in such a way that the basis flow is rotating about the $z$-axis. The angular velocity $\epsilon$, relative to the basic angular velocity, of the precession is assumed to be constant and serves as a measure for strength of the Coriolis force. The velocity components of the flow to be considered is $\boldsymbol{U}(x, y, z)=$ $(-(1-\delta) y,(1+\delta) x,-2 \epsilon y)$, which is linear in coordinates. This is a steady solution of the Navier-Stokes equation supplemented with the Coriolis force for small values of $|\delta|$ and $|\epsilon|,{ }^{9}$ and is so even for an arbitrary value of $\epsilon$ in case $\delta=0$. Here the constant $(-1<\delta<1)$ is a measure for the ellipticity. Incidentally Kerswell ${ }^{8}$ considered the basic flow $\boldsymbol{U}(x, y, z)=(-y, x-2 \epsilon z, 0)$ 
for precessing motion of the fluid with the same precessional angular velocity $\boldsymbol{\Omega}=(\epsilon, 0,0)$. The difference lies in the pressure distribution $p(x, y, z)$. In our case, ${ }^{9} p=\left(x^{2}+y^{2}\right) / 2-2 \epsilon^{2} y^{2}$, while in the latter case, the pressure varies along the $z$ axis as $p=\left(x^{2}+y^{2}\right) / 2+2 \epsilon^{2} z^{2}-2 \epsilon x z$. The boundary conditions will favor a realizable local field. Owing to the linear dependence of the velocity profile, of the basic flow, in coordinates, we may superimpose the following form of three-dimensional localized disturbance velocity $\boldsymbol{u}^{\prime}$ and pressure $p^{\prime}$

$$
\left[\boldsymbol{u}^{\prime}(\boldsymbol{x}, t), p^{\prime}(\boldsymbol{x}, t)\right]=\exp (\mathrm{i} \boldsymbol{k}(t) \cdot \boldsymbol{x})[\boldsymbol{a}(t), b(t)],
$$

on the basic flow, where $\boldsymbol{k}(t)$ is the time dependent of wave vector. ${ }^{1}$ We are concerned with amplification or non-amplification of the amplitude $\boldsymbol{a}$ and $b$. These are substituted into the Euler equations and terms quadratic in disturbance amplitude are neglected. Then the linearized Euler equations are reduced to a coupled system of characteristic equations:

$$
\begin{gathered}
\frac{\mathrm{d} \boldsymbol{k}}{\mathrm{d} t}=-\mathcal{L}^{T} \boldsymbol{k}, \\
\frac{\mathrm{d} \boldsymbol{a}}{\mathrm{d} t}=\left(\frac{2 \boldsymbol{k} \boldsymbol{k}^{T}}{k^{2}}-I\right) \mathcal{L} \boldsymbol{a}-2\left(\boldsymbol{\Omega} \times \boldsymbol{a}-[(\boldsymbol{\Omega} \times \boldsymbol{a}) \cdot \boldsymbol{k}] \frac{\boldsymbol{k}}{k^{2}}\right),
\end{gathered}
$$

together with the incompressibility condition

$$
\boldsymbol{k} \cdot \boldsymbol{a}=0,
$$

where $\boldsymbol{I}$ is the $3 \times 3$ unit matrix, superscript $T$ stands for taking the transpose, and $\mathcal{L}$ is a $3 \times 3$ constant matrix defined by

$$
\mathcal{L}=\left[\frac{\partial U_{i}}{\partial x_{j}}\right]=\left[\begin{array}{ccc}
0 & -(1-\delta) & 0 \\
1+\delta & 0 & 0 \\
0 & -2 \epsilon & 0
\end{array}\right]
$$

Equation (2) has the following general solution:

$$
\boldsymbol{k}=\left(\sin \theta \cos \beta t-\frac{2 \epsilon \cos \theta}{1-\delta}, \sqrt{\frac{1-\delta}{1+\delta}} \sin \theta \sin \beta t, \cos \theta\right),
$$

where $\theta$ is a constant close to the inclination angle of the wave vector from the $z$-axis, and $\beta=1-\delta^{2}$.

With this form, eq. (3) becomes a matrix Floquet problem. For handling these characteristic equations, it is advantageous to employ variables introduced in ref. 10 that reduce eqs. (2)-(4) to a coupled system of Hill-Schrödinger equations with period $2 \pi / \beta$. We extend their treatment for two-dimensional basic flows to three-dimensional one. We decompose vectors into a vector component in the $x y$-plane denoted by the $\perp$ subscript and a scalar component along the z-axis denoted by the $\|$ subscript and write

$$
\boldsymbol{U}=\left[\begin{array}{c}
\boldsymbol{U}_{\perp} \\
-2 \epsilon y
\end{array}\right], \quad \mathcal{L}=\left[\begin{array}{cc}
\mathcal{L}_{\perp} & \mathbf{0} \\
-2 \epsilon & 0
\end{array}\right]
$$


Under this restriction, eq. (2) decomposes into

$$
\mathrm{d} \boldsymbol{k}_{\perp} / \mathrm{d} t=-\mathcal{L}_{\perp}^{T} \boldsymbol{k}_{\perp}+2 \epsilon k_{2}, \quad \mathrm{~d} k_{\|} / \mathrm{d} t=0 .
$$

The parallel component $k_{\|}$of $\boldsymbol{k}$ is constant and the perpendicular component $\boldsymbol{k}_{\perp}$ of $\boldsymbol{k}$ decouples from the parallel component.

Let us write the basic vorticity as $\mathbf{W}=\left(w_{1}, w_{2}, w_{3}\right)=\nabla \times \boldsymbol{U}$. Denote the amplitude of the disturbance vorticity to be $\boldsymbol{b}=\boldsymbol{k} \times \boldsymbol{a}$ and introduce new variables

$$
p=\frac{|\boldsymbol{k}|}{\left|\boldsymbol{k}_{\perp}\right|} \boldsymbol{k}_{\perp} \cdot \boldsymbol{a}_{\perp}, \quad q=\frac{|\boldsymbol{k}|}{\left|\boldsymbol{k}_{\perp}\right|} \boldsymbol{b}_{\|} \cdot
$$

Then eqs. (2) and (3) are reduced to

$$
\frac{\mathrm{d}}{\mathrm{d} t}\left[\begin{array}{l}
p \\
q
\end{array}\right]=\left[\begin{array}{cc}
A & B \\
C & -A
\end{array}\right]\left[\begin{array}{l}
p \\
q
\end{array}\right],
$$

where the matrix entries are

$$
\begin{aligned}
& A=\frac{\mathrm{d}}{\mathrm{d} t} \log \frac{\left|\boldsymbol{k}_{\perp}\right|}{|\boldsymbol{k}|}-2 \epsilon \frac{k_{2} k_{3}}{k_{\perp}^{2}}, \\
& B=\frac{2 k_{3}^{2}}{k^{2} k_{\perp}^{2}} \boldsymbol{k}_{\perp}^{T} \mathcal{H} \boldsymbol{k}_{\perp}+2 \epsilon \frac{k_{1} k_{3}^{2}}{k^{2} k_{\perp}^{2}}, \\
& C=\frac{-2 \epsilon k_{1}\left(2 k_{\perp}^{2}+k_{3}^{2}\right)}{k_{\perp}^{2} k_{3}}-\frac{k_{1}}{k_{3}} w_{1}-w_{3},
\end{aligned}
$$

supplemented by

$$
\mathcal{H}=\mathcal{L}_{\perp}\left[\begin{array}{cc}
0 & 1 \\
-1 & 0
\end{array}\right] .
$$

To get the desired Schrödinger equation, we pose

$$
\begin{aligned}
& p(t)=P(t) \exp \left(\frac{1}{2} \int_{0}^{t} \frac{\dot{B}\left(t^{\prime}\right)}{B\left(t^{\prime}\right)} \mathrm{d} t^{\prime}\right), \\
& q(t)=Q(t) \exp \left(\frac{1}{2} \int_{0}^{t} \frac{\dot{C}\left(t^{\prime}\right)}{C\left(t^{\prime}\right)} \mathrm{d} t^{\prime}\right),
\end{aligned}
$$

with a dot designating the differentiation in $t$. Substituting eqs. (11) and (12) in eq. (10), we obtain Hill-Schrödinger equations:

$$
\begin{aligned}
& \frac{\mathrm{d}^{2} P(t)}{\mathrm{d} t^{2}}+\hat{\beta}_{P}(t) P(t)=0, \\
& \frac{\mathrm{d}^{2} Q(t)}{\mathrm{d} t^{2}}+\hat{\beta}_{Q}(t) Q(t)=0,
\end{aligned}
$$

where

$$
\begin{aligned}
& \hat{\beta}_{P}(t)=-\frac{\dot{B}^{2}}{4 B^{2}}+\frac{\dot{B}}{2 B}-\dot{A}+A \frac{\dot{B}}{B}-B C-A^{2}, \\
& \hat{\beta}_{Q}(t)=-\frac{\dot{C}^{2}}{4 C^{2}}+\frac{\dot{C}}{2 C}+\dot{A}-A \frac{\dot{C}}{C}-B C-A^{2} .
\end{aligned}
$$

The detail of calculation is referred to refs. 10 and 11. 


\section{Influence of the precession on a rotating flow with circular streamlines}

At the outset, we examine the influence of the Coriolis force only. For this case, the basic flow can be rewritten as $\boldsymbol{U}(x, y, z)=(-y, x,-2 \epsilon y)$. This undisturbed steady state has circular streamlines in the $x y$ plane, and, with the restriction of $\delta=0$, is a solution of the NavierStokes equations for arbitrary precession rate $\epsilon$. We treat separately the cases of $|\epsilon| \ll 1$ and $|\epsilon| \gg 1$.

\subsection{The case of small precession frequency}

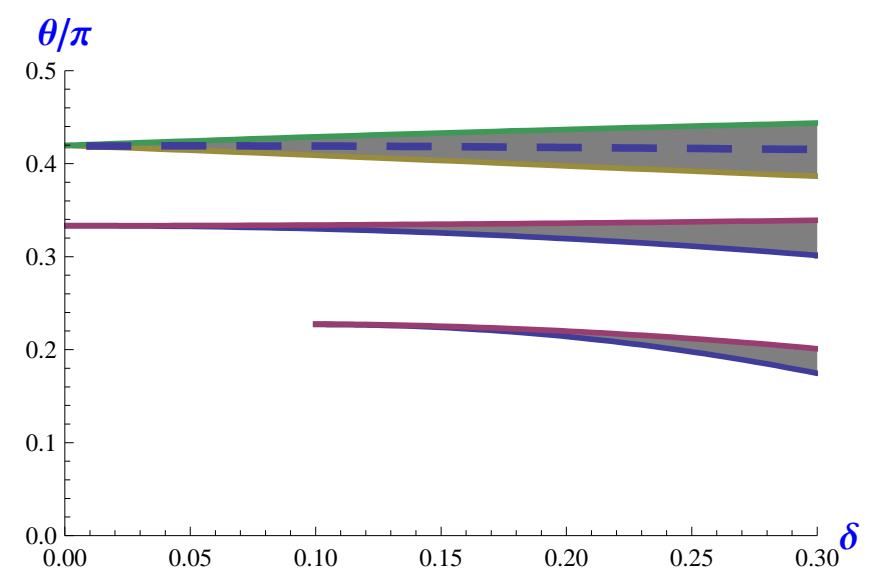

Fig. 1. Instability regions, in the $(\epsilon, \theta)$ plane, calculated from eq. (10) for small values of $\epsilon(0 \leq \epsilon \leq$ $0.3)$, in the absence of ellipticity $(\delta=0)$. The instability occurs in the gray region. For given $\epsilon$, the maximum growth rate is taken at the inclination angle $\theta$ shown by the thick solid line.

First we work out the temporal evolution of disturbances for the case of a weak external Coriolis force $(|\epsilon|<1)$. The Floquet method is directly applicable to our equation. We calculate the Floquet exponent and the stability boundary in the parameter space $(\epsilon, \theta)$ from eq. (10) both numerically and analytically. Figure 1 summarizes the numerical result for a weak external Coriolis force $(\epsilon \leq 0.3)$, with $\delta=0$. The instability occurs in the gray regions between two solid lines. The three instability bands are identified. The growth rates depend on the strength $\epsilon$ of the external Coriolis force. For a given $\epsilon$, as far as it is smaller than a certain value $(\epsilon \lesssim 0.56)$ as will be discussed later, the largest growth rate occurs at the value of the inclination angle $\theta$ in the midway in the dominant instability region emanating from $\theta=\cos ^{-1}(1 / 4) \approx 1.3181161$, or $\theta / \pi \approx 0.41956938$, which is displayed by the thick dashed line.

For very small values of $|\epsilon|$, eq. (10) is approximated by the Mathieu equation. The Mathieu equation describes the small amplitude oscillations of a pendulum whose length changes periodically in time. If the length changes at a certain frequency then the amplitude of the pendulum will grow in time, a phenomenon called parametric excitation. The Mathieu equation 
is written, for the natural frequency $\omega$ and the forcing frequency $\nu$, as

$$
\ddot{\varphi}+\omega^{2}(1+\gamma \cos \nu t) \varphi=0,
$$

where the perturbation amplitude $\gamma$ is assumed to be small.

For the case $|\epsilon| \ll 1, \mathrm{~A}, \mathrm{~B}$ and $\mathrm{C}$ in eq. (10) are expanded in a power series in $\epsilon$, giving, to first order,

$$
\begin{gathered}
A \approx-\epsilon \sin t \sin 2 \theta, \\
B \approx 2 \cos ^{2} \theta-2 \epsilon \cos ^{2} \theta \cos t(-3+2 \cos 2 \theta) \cot \theta, \\
C \approx-2-4 \epsilon \cos t \csc 2 \theta .
\end{gathered}
$$

Equation (10) gives way to the Mathieu equation

$$
\ddot{q}+\left[4 \cos ^{2} \theta-\frac{1}{2} \epsilon\left(1-4 \cos ^{2} \theta-44 \cos ^{4} \theta+32 \cos ^{6} \theta\right) \csc \theta \sec \theta \cos t\right] q=0 .
$$

Comparison of eq. (16) with eq. (15) tells that $\nu=1, \omega^{2}=4 \cos ^{2} \theta$ and that $\gamma$ is found from

$$
\omega^{2} \gamma=-\frac{1}{2} \epsilon\left(1-4 \cos ^{2} \theta-44 \cos ^{4} \theta+32 \cos ^{6} \theta\right) \csc \theta \sec \theta .
$$

According to the Floquet theory as applied to (15), parametric resonance instability may be admitted only around the parameter region where $\omega / \nu$ is an integer or a half integer. This necessary condition for instability reads, in the present context, $\omega^{2}=4 \cos ^{2} \theta=(n / 2)^{2}(n=$ $1,2,3, \ldots)$, among which the subharmonic resonance $\cos \theta= \pm 1 / 4$ is the primary instability mode.

By an asymptotic analysis of eq. (16) with use of the Mathieu method, ${ }^{12}$ the growth rate at $\cos \theta= \pm 1 / 4$ is manipulated, to $O(\epsilon)$, to be $\sigma \approx 5 \sqrt{15}|\epsilon| / 16$. Notably, the angle $\cos ^{-1}( \pm 1 / 4)$ and the factor $5 \sqrt{15} / 16$ in the growth rate coincide with those for the primary local instability mode of a thin vortex ring. ${ }^{13}$ The Mathieu method will be expounded in the following section $(\S 4)$, where this asymptotic result of eq. (16) is touched upon.

\subsection{The case of large precession frequency}

We extend the numerical calculation of eq. (10) to large values of $|\epsilon|$, with keeping no ellipticity $(\delta=0)$. For a large value of $|\epsilon|$, the instability region covers almost the whole range of $\theta(0 \leq \theta \leq \pi / 2)$ as shown with hatch in Fig. 2. As $\epsilon$ is increased without bound, the maximum growth rate $\sigma / \epsilon$ increases and appears to tend, in the limit of $|\epsilon| \rightarrow \infty$, to

$$
\sigma=1
$$

Concomitantly, the inclination angle corresponding to the maximum growth rate approaches $\theta=0$.

For the case of a strong external Coriolis force $(|\epsilon| \gg 1)$, it is reasonable to calculate the growth rate analytically by prescribing $\theta=0$. In this case, eq. (2) yields

$$
\boldsymbol{k}=(-2 \epsilon, 0,1),
$$


J. Phys. Soc. Jpn.

Full PAPER

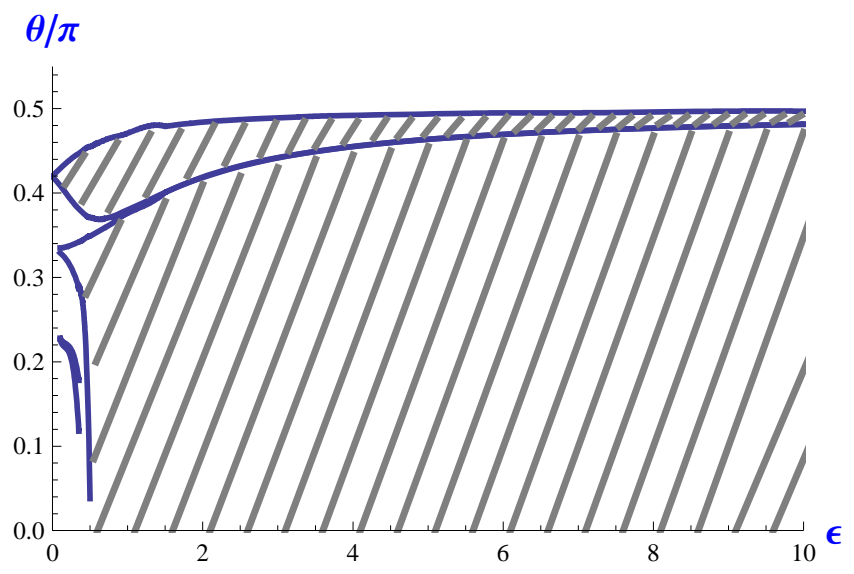

Fig. 2. Instability regions, in the $(\epsilon, \theta)$ plane, calculated from eq. (10) over a wide range of $\epsilon$, in the absence of ellipticity $(\delta=0)$. The instability occurs in the hatched region.

and then eq. (10) becomes

$$
\frac{\mathrm{d}}{\mathrm{d} t}\left[\begin{array}{l}
p \\
q
\end{array}\right]=\left[\begin{array}{cc}
0 & \frac{1}{1+4 \epsilon^{2}} \\
4 \epsilon^{2}-1 & 0
\end{array}\right]\left[\begin{array}{l}
p \\
q
\end{array}\right] .
$$

This is solved at once to produce, in the limit of $|\epsilon| \rightarrow \infty$, eq. (17). When the inclination angle is restricted to $\theta=0$, the critical value of the precession angular velocity for instability is $|\epsilon|=0.5$. At this critical value, $q=$ const. and $p$ grows linear in $t$. This result is confirmed from Fig. 3 which depicts the maximum growth rate for a given $\epsilon$.

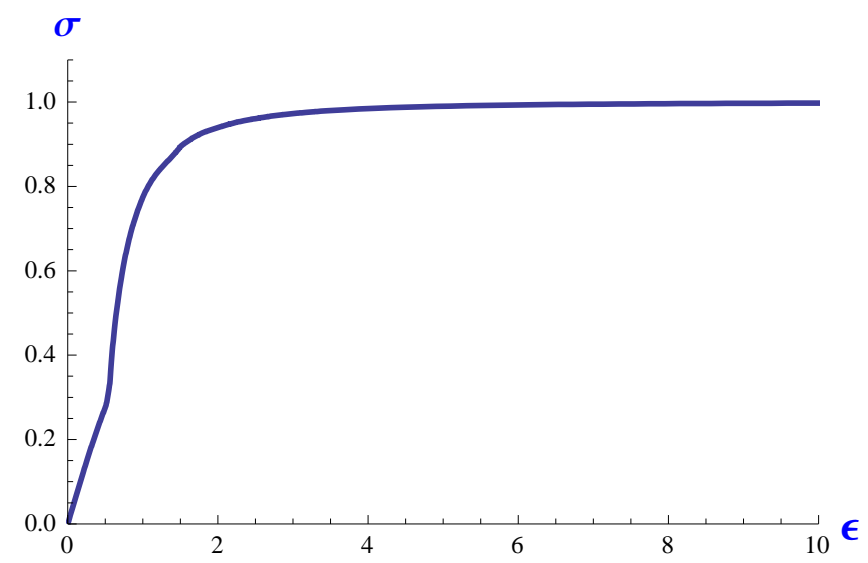

Fig. 3. Maximum instability growth rate as a function of $\epsilon$ in the absence of ellipticity $(\delta=0)$,

There are three instability bands as is observed from Fig. 1. For very small values of $\epsilon$, the band issuing from $\theta=\cos ^{-1}(1 / 4)$ predominates. As $|\epsilon|$ becomes larger, this is superseded by the second band and then by the third band, and for $\epsilon>0.56$, the dominant mode occurs 


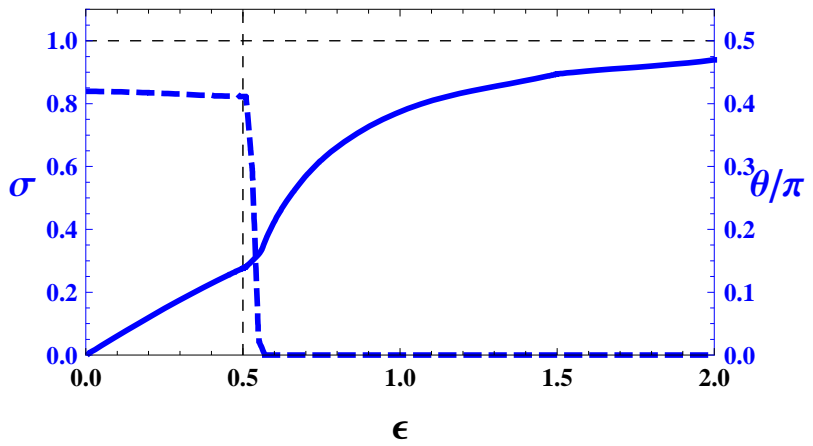

Fig. 4. Variation of the maximum growth rate with $\epsilon$ for $\delta=0$. The solid line is the maximum growth rate $\sigma$ and the dashed line is the corresponding inclination angle $\theta$ as functions of $\epsilon$.

at $\theta=0$. This exchange of role by the instability bands is shown in Fig. 4 . The dashed line draws the angle $\theta$ at which the growth rate attains its maximum, for a given value of $\epsilon$.

\section{Combined influence of the precession and the straining field}

Now we are ready to tackle with the combined effects of the Coriolis force and the elliptical straining field in our model. To search for possible instabilities over a wide range of the parameter space $(\epsilon, \delta)$ is rather cumbersome. Here instead, we focus our attention on cases where $|\epsilon|$ and $|\delta|$ are both small, in particular

(1) modification of the elliptical instability by the Coriolis effect,

(2) modification of the Coriolis instability by the straining field.

We are concerned with the case where the both parameters $|\epsilon|$ and $|\delta|$ are small. Equation (10), when expanded in powers of $\epsilon$ and $\delta$, reduces to the Hill equation which is a generalization of the Mathieu equation with its potential augmented by second harmonics of the fundamental frequency:

$$
\ddot{q}+\left(u+2 \epsilon a_{1} \cos t+2 \delta a_{2} \cos 2 t\right) q=0,
$$

where

$$
\begin{gathered}
u(\theta)=\cos ^{2} \theta\left(4+4 \delta \sin ^{2} \theta\right), \\
a_{1}(\theta)=-\frac{1}{4}\left(1-4 \cos ^{2} \theta-44 \cos ^{4} \theta+32 \cos ^{6} \theta\right) \csc \theta \sec \theta, \\
a_{2}(\theta)=-\cos ^{2} \theta\left(2 \sin ^{2} \theta+3\right) .
\end{gathered}
$$

We rely on the Mathieu method ${ }^{12}$ to find parametric resonance and to calculate its growth rate.

Case (1)

First we assume the solution $q$ of eq. (19) in the following form:

$$
q=e^{\sigma t}\left(\ldots+b_{-2} e^{-2 \mathrm{i} t}+b_{-1} e^{-\mathrm{i} t}+b_{0}+b_{1} e^{\mathrm{i} t}+b_{2} e^{2 \mathrm{i} t}+\ldots\right) .
$$


Upon substitution, eq. (19) gives way to Hill's determinantal matrix form:

$$
\left[\begin{array}{ccccc}
c_{1} & \epsilon a_{1} & \delta a_{2} & 0 & 0 \\
\epsilon a_{1} & c_{2} & \epsilon a_{1} & \delta a_{2} & 0 \\
\delta a_{2} & \epsilon a_{1} & c_{3} & \epsilon a_{1} & \delta a_{2} \\
0 & \delta a_{2} & \epsilon a_{1} & c_{4} & \epsilon a_{1} \\
0 & 0 & \delta a_{2} & \epsilon a_{1} & c_{5}
\end{array}\right]\left[\begin{array}{c}
b_{-2} \\
b_{-1} \\
b_{0} \\
b_{1} \\
b_{2}
\end{array}\right]=\mathbf{0}
$$

where

$$
c_{1}=(\sigma-2 \mathrm{i})^{2}+u, c_{2}=(\sigma-\mathrm{i})^{2}+u, c_{3}=\sigma^{2}+u, c_{4}=(\sigma+\mathrm{i})^{2}+u, c_{5}=(\sigma+2 \mathrm{i})^{2}+u .
$$

In order for a non-trivial solution $\left(b_{-2}, b_{-1}, b_{0}, b_{1}, b_{2}\right) \neq \mathbf{0}$ to be admitted, the determinant of the matrix in eq.(20) must be zero. When $\delta=\epsilon=0$, the eigenvalue $\sigma$ is obtained at once as:

$$
\sigma^{2}=-u,-(\sqrt{u} \pm 1)^{2},-(\sqrt{u} \pm 2)^{2},
$$

where $-(\sqrt{u} \pm 2)^{2}$ and $-(\sqrt{u} \pm 1)^{2}$ have multiplicity two in $\sigma^{2}$. We look into how these eigenvalues are moved when the precession $(\epsilon \neq 0)$ and the straining field $(\delta \neq 0)$ are called into play. When the parameters $\epsilon$ and $\delta$ are small in magnitude, the instability is possible only around $u=0,1$ and 4 .

(a) Case of $\delta \neq 0$ and $\epsilon=0$

First let us switch off the Coriolis force $(\epsilon=0)$. Considering the effect of small ellipticity to $\mathrm{O}\left(\delta^{2}\right)$ and, if necessary, a higher order, we have

(i) at $u=0, \sigma^{2}=-\frac{1}{2}\left(\delta a_{2}\right)^{2}$,

(ii) at $u=1$ or $\cos \theta= \pm \frac{1}{2}\left(1-\frac{3}{8} \delta\right), \sigma^{2}=\frac{1}{4}\left(\delta a_{2}\right)^{2}$ or $\sigma \approx \frac{9|\delta|}{16}\left(1-\frac{2 \delta}{3}\right)$,

(iii) at $u=4, \sigma^{2}=\mathrm{o}\left(\delta^{10}\right)$.

No instability emerges at $u=0$. At $u=4$, the growth rate is likely to be $\sigma^{2}=0$, indicative of marginal stability, since $\sigma^{2}=0$ to as high as $\mathrm{O}\left(\delta^{10}\right)$. The instability occurs only at $u=1$ for which $\cos \theta \approx \pm 1 / 2$ or $\theta=\pi / 3$ and $2 \pi / 3$, and its growth rate is $\sigma \approx 9|\delta| / 16$, a well known result of the elliptical instability. ${ }^{1,2}$

(b) Case of $\delta=0$ and $\epsilon \neq 0$

In this case, we consider the effect only of the small Coriolis force to $\mathrm{O}\left(\epsilon^{4}\right)$, taking no account of straining effect $(\delta=0)$.

(i) at $u=0, \sigma^{2}=-2\left(\epsilon a_{1}\right)^{2}-\frac{25}{2}\left(\epsilon a_{1}\right)^{4}$,

(ii) at $u=1$ or $\cos \theta= \pm \frac{1}{2}, \sigma^{2}=\frac{5}{36}\left(\epsilon a_{1}\right)^{4}$ or $\sigma \approx \frac{9 \sqrt{5}}{32} \epsilon^{2}$,

(iii) at $u=4, \sigma^{2}=-\frac{1}{144}\left(\epsilon a_{1}\right)^{4}$.

Instability comes out at $u=1$ or $\theta=\pi / 3$ and $2 \pi / 3$ with growth rate $\sigma \approx 9 \sqrt{5} \epsilon^{2} / 32$, being second order in $\epsilon$. This mode corresponds to the middle instability band issuing from about $\theta \approx \pi / 3$ in Fig. 1. This band is narrower than the band of the subharmonic instability which 
is issuing from $\theta=\cos ^{-1}(1 / 4)$ with growth rate $\sigma=\mathrm{O}(|\epsilon|)$, as will be calculated subsequently.

(c) Case of $\delta \neq 0$ and $\epsilon \neq 0(|\epsilon| \ll|\delta|)$

Next we turn to the influence of the slow precession, to $\mathrm{O}\left(\epsilon^{2}\right)$, upon the elliptical instability.

(i) at $u=0, \sigma^{2}=-2\left(\epsilon a_{1}\right)^{2}+\frac{\left(\delta a_{2}\right)^{2}}{4}\left(-2-\frac{33}{4}\left(\epsilon a_{1}\right)^{2}\right)$,

(ii) at $u=1, \sigma^{2}=\frac{\left(\delta a_{2}\right)^{2}}{4}\left(1-\frac{\left(\epsilon a_{1}\right)^{2}}{9}\right)$ or $\sigma \approx \frac{9|\delta|}{16}\left(1-\frac{2 \delta}{3}-\frac{3 \epsilon^{2}}{32}\right)$,

(iii) at $u=4, \sigma^{2}=-\frac{1}{288}\left(\epsilon a_{1}\right)^{2}\left[3\left(\delta a_{2}\right)^{2}+2\left(\epsilon a_{1}\right)^{2}\right]$.

No instability is invited to $\mathrm{O}\left(\delta^{2}\right)$ and $\mathrm{O}\left(\delta^{2}, \epsilon^{2}\right)$ even if the Coriolis force is included. Rather the Coriolis force, regardless of whether the precession frequency $\epsilon$ is either positive or negative, acts to reduce the growth rate of the elliptical instability around $\theta=\pi / 3$ and $2 \pi / 3$.

Case (2)

Next, we seek the solution of eq. (19) in the following form: ${ }^{12}$

$$
q=e^{\sigma t}\left(\ldots+b_{-\frac{3}{2}} e^{-\frac{3}{2} \mathrm{i} t}+b_{-\frac{1}{2}} e^{-\frac{1}{2} \mathrm{i} t}+b_{\frac{1}{2}} e^{\frac{1}{2} \mathrm{i} t}+b_{\frac{3}{2}} e^{\frac{3}{2} \mathrm{i} t} \ldots\right) .
$$

With this form, eq. (19) is converted to Hill's determinantal matrix form:

where

$$
\left[\begin{array}{cccc}
d_{1} & \epsilon a_{1} & \delta a_{2} & 0 \\
\epsilon a_{1} & d_{2} & \epsilon a_{1} & \delta a_{2} \\
\delta a_{2} & \epsilon a_{1} & d_{3} & \epsilon a_{1} \\
0 & \delta a_{2} & \epsilon a_{1} & d_{4}
\end{array}\right]\left[\begin{array}{c}
b_{-\frac{3}{2}} \\
b_{-\frac{1}{2}} \\
b_{\frac{1}{2}} \\
b_{\frac{3}{2}}
\end{array}\right]=\mathbf{0}
$$

$$
d_{1}=\left(\sigma-\frac{3}{2} \mathrm{i}\right)^{2}+u, d_{2}=\left(\sigma-\frac{1}{2} \mathrm{i}\right)^{2}+u, d_{3}=\left(\sigma+\frac{1}{2} \mathrm{i}\right)^{2}+u, d_{4}=\left(\sigma+\frac{3}{2} \mathrm{i}\right)^{2}+u
$$

In the absence of the precession and the strain $(\delta=\epsilon=0)$, the requirement of vanishing determinant of the matrix in eq.(21) provides the eigenvalues

$$
\sigma^{2}=-\left(\sqrt{u} \pm \frac{1}{2}\right)^{2},-\left(\sqrt{u} \pm \frac{3}{2}\right)^{2}
$$

all having multiplicity two in $\sigma^{2}$. For small values of $|\epsilon|$ and $|\delta|$, the instability is possible only around $u=1 / 4$ and $9 / 4$.

(a) Case of $\epsilon \neq 0$ and $\delta=0$

First we consider the effect of only the small Coriolis force to $\mathrm{O}\left(\epsilon^{4}\right)$, leaving out the straining effect $(\delta=0)$.

(i) at $u=\frac{1}{4}$ or $\cos \theta= \pm \frac{1}{4}, \sigma^{2}=\left(\epsilon a_{1}\right)^{2}-\frac{15}{4}\left(\epsilon a_{1}\right)^{4}$ or $\sigma \approx \frac{5 \sqrt{15}}{32}|\epsilon|\left(1-\frac{5625}{8192} \epsilon^{2}\right)$,

(ii) at $u=\frac{9}{4}, \sigma^{2}=-\frac{1}{36}\left(\epsilon a_{1}\right)^{4}$,

the latter being neutrally stable. The former is the subharmonic instability and is the primary mode of precession origin, that is, $\sigma \approx 5 \sqrt{1} 5|\epsilon| / 32$ at $\cos \theta= \pm 1 / 4$, which was discussed at length in $\S 3.1$. 
(b) Case of $\epsilon=0$ and $\delta \neq 0$

Next let us switch off the Coriolis force $(\epsilon=0)$, and consider the effect of only small ellipticity to a higher order.

(i) at $u=\frac{1}{4}, \sigma^{2}=-\frac{1}{4}\left(\delta a_{2}\right)^{4}$,

(ii) at $u=\frac{9}{4}, \sigma^{2}=-\frac{1}{36}\left(\delta a_{2}\right)^{4}$.

No instability occurs at $u=1 / 4$ and $u=9 / 4$ as has been known since the pioneering work on the elliptical instability. ${ }^{1}$

(c) Case of $\delta \neq 0$ and $\epsilon \neq 0(|\delta| \ll|\epsilon|)$

Now incorporating the influence of the weak elliptical strain into the precessional instability, we have

(i) at $u=\frac{1}{4}, \sigma^{2}=\left(\epsilon a_{1}\right)^{2}\left(1+2 \delta a_{2}\right)$ or $\sigma \approx \frac{5 \sqrt{15}|\epsilon|}{32}\left(1+\frac{669}{640} \delta\right)$,

(ii) at $u=\frac{9}{4}, \sigma^{2}=-\frac{1}{36}\left[\left(\delta a_{2}\right)^{2}-\left(\epsilon a_{1}\right)^{2}\right]^{2}$.

The growth rate of the precessional instability becomes larger or smaller depending on the orientation of the major axis. The precessional instability is weakened if the precession axis is oriented to the major axis $(\delta<0)$, but is enhanced if the precession axis is oriented to the minor axis $(\delta>0)$.

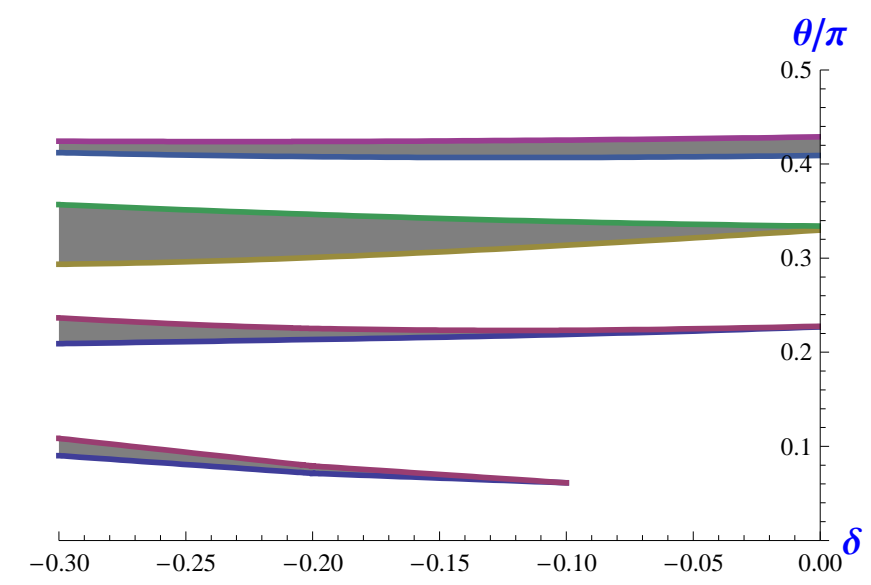

Fig. 5. Instability regions for the cooperative action of the Coriolis force and the straining field in the $(\delta, \theta)$ plane, with fixing $\epsilon=0.1$. The instability occurs in the gray regions. The precession axis is parallel to the major axis of elliptical streamlines $(\delta \leq 0)$.

We calculate the stability boundary for the cooperative action of the Coriolis force and the elliptically straining field from eq. (10), with varying the ellipticity parameter $\delta$, while fixing $\epsilon=0.1$. The cases of $\delta \leq 0$ and $\delta \geq 0$ are shown in Figs. 5 and 6 respectively. The instability occurs in the gray region. Without precession, only the single band of the elliptical instability exists but the precession adds two instability bands for small $|\delta|$ and three bands for $|\delta|$ larger 


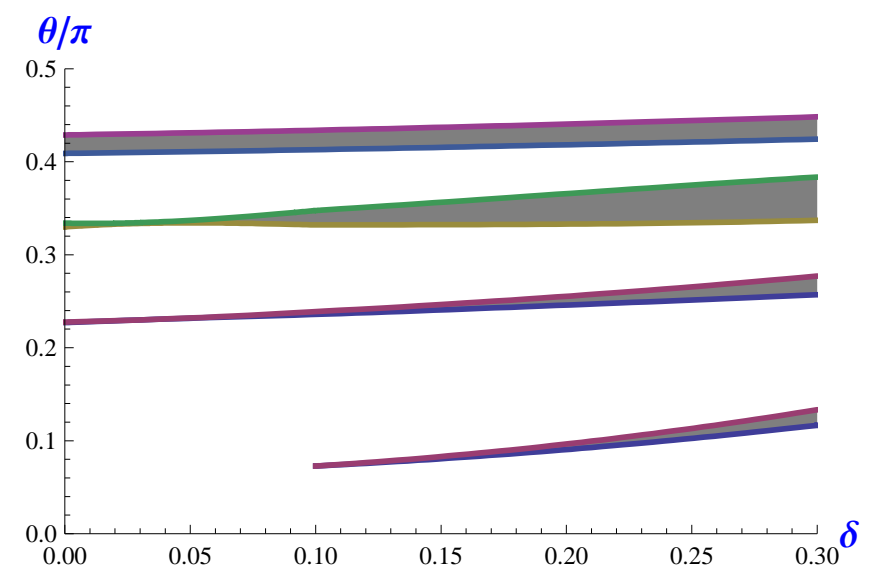

Fig. 6. The same as Fig. 5, but the precession axis is parallel to the minor axis of elliptical streamlines $(\delta \geq 0)$.

than about 0.1 . The case of $\delta=0$ restores Fig. 1 with $\epsilon=0.1$. In Figs. 5 and 6 , the dominant bands are the ones issuing from $\theta=\cos ^{-1}(1 / 4)(\theta / \pi \approx 0.41956938)$ and $\theta=\cos ^{-1}(1 / 2)$ $(\theta / \pi=1 / 3)$. The former is the subharmonic mode of the precessional origin and the latter consists of the subharmonic mode of the strain origin, or the elliptical instability, and the fundamental mode of the precessional origin. Comparing the uppermost instability band at $|\delta|=0.3$ between Figs. 5 and 6 , we observe that the band is wider for $\delta>0$ (Fig. 6) than for $\delta<0$ (Fig. 5), in keeping with the above asymptotic analysis of using the Mathieu method; the growth rate increases with $\delta$ and, as a consequence, the uppermost band broadens with $\delta$. According to our asymptotic analysis, the growth rate of the elliptical instability is made smaller by the precession. Correspondingly, the instability band around $\theta=\pi / 3$ is narrower than the case of single influence of the straining field. ${ }^{1}$ The third band from above, being thin, emanates from the angle about $\theta=\cos ^{-1}(3 / 4)$, or $\theta / \pi \approx 0.23005346$, and is attributed to the third subharmonic mode of the precessional origin. Moreover, the fourth band makes its appearance at small inclination angle $\theta$ for $|\delta|$ larger than a certain value about 0.1 . This is a mode born genuinely by a combined action of the precession and the straining field.

\section{Conclusions}

We have studied the stability of a precessing flow with respect to short-wave threedimensional disturbances by adopting the geometrical optics technique. Influence of elliptical deformation of streamlines has been examined as well. This technique converts the partial differential equations of hydrodynamic stability into a system of ordinary differential equations, or the characteristic equations, associated with motion of fluid particles. In the case of a basic flow with closed streamlines, these equations have coefficients periodic in time, and are amenable to the Floquet theory. The resulting matrix eigenvalue problem is solved numerically. For small values of the precession frequency $|\epsilon|$ and ellipticity $|\delta|$, the set of the 
characteristic equations are reduced to the Mathieu equation for the case of $\delta=0$ and to a simple form of the Hill equation for the case of $\delta \neq 0$. The Floquet exponent is manipulated in the form of a power series in $\epsilon$ and $\delta$ by means of the Mathieu method.

We have proceeded in three steps. In the first step, we set the basic flow to be close to rigid body rotation subject to a weak external Coriolis force $|\epsilon| \ll 1$. The disturbance equations are reducible to the standard form of the Mathieu equation. The instability mode shares a common mathematical structure with the curvature instability of a vortex ring. ${ }^{13,14}$ In the second step, we enter the regime of strong external Coriolis force. A new instability mode, with its growth rate given by the angular velocity of the basic rotation, has been disclosed. The third step addresses the influence of cooperative action of the Coriolis force and the elliptically straining field with both $|\epsilon|$ and $|\delta|$ taken to be small. The Mathieu method captures the onset of the instability, and the effect of the precession on the elliptical instability and the effect of the reverse action. These findings have been substantiated by numerical calculation.

Recently mathematical machinery to handle nonlinear evolution of disturbance has been developed for the elliptical instability ${ }^{15-17}$ and for the precessional instability with no strain. ${ }^{18,19}$ The nonlinear interaction of disturbances, the modification of mean flow by the boundary layer and the viscous dissipation drastically alter the evolution of linearly excited waves. The nonlinear excitation of waves via the secondary and the tertiary instability brings in chaotic behavior of rotating flows and in transition to turbulence. ${ }^{15-18}$ In some cases, a weakly nonlinear analysis is capable of describing a saturated state. ${ }^{19}$ This paper suggests a rich behavior of unstable waves on a rotating flow acted by a combination of the precession and the strain field. The nonlinear stage of evolution of excited waves calls for an individual investigation.

\section{Acknowledgment}

We would like to thank Mr. T. Futae, M. Adachi and Y. Mie for helpful comments. M. M. N. would like to express her deep appreciation to the Ministry of Education, Culture, Sports, Science and Technology, Japan for financial support with scholarship award. Y. F. was supported in part by a Grant-in-Aid for Scientific Research from the Japan Society of Promotion of Science (No. 21540390). 


\section{References}

1) B. J. Bayly: Phys. Rev. Lett. 57 (1986) 2160.

2) F. Waleffe: Phys. Fluids A 2, (1990) 76.

3) A. Lifschitz and E. Hameiri: Phys. Fluids A 3 (1991) 2644.

4) S. Friedlander and M. M. Vishik: Phys. Rev. Lett. 66 (1991) 2204.

5) A. D. D. Craik: J. Fluid Mech. 198 (1989) 275.

6) T. Miyazaki: Phys. Fluids A 5 (1993) 2702.

7) Y. Fukumoto and T. Miyazaki: J. Phys. Soc. Jpn. 65 (1996) 107.

8) R. R. Kerswell: Geophys. Astrophys. Fluid Dyn. 72 (1993) 107.

9) A. Mahalov: Phys. Fluids 5 (1993) 891.

10) B. J. Bayly, D. D. Holm and A. Lifschitz: Philos. Trans. R. Soc. Lond. A 354 (1996) 895.

11) A. Lifschitz: Advances in Applied Mathematics 15 (1994) 404.

12) E. L. Ince: Ordinary Differential Equations (Dover Publications, New York, 1956) p. 384.

13) Y. Hattori and Y. Fukumoto: Phys. Fluids 15 (2003) 3151.

14) Y. Fukumoto and Y. Hattori: J. Fluid Mech. 526 (2005) 77.

15) D. M. Mason and R. R. Kerswell: J. Fluid Mech. 396 (1999) 73.

16) Y. Fukumoto, Y. Hattori and K. Fujimura: in Proc. 3rd Int. Conf. Vortex Flows and Vortex Models, ed. K. Kamemoto (Japan Society of Mechanical Engineers, 2005) p. 149.

17) S. B. Rodrigues and J. D. Luca: Phys. Fluids 21 (2009) 014108.

18) D. M. Mason and R. R. Kerswell: J. Fluid Mech. 471 (2002) 71.

19) P. Meunier, C. Eloy, R. Lagrange and F. Nadal: J. Fluid Mech. 599 (2008) 405. 


\section{List of MI Preprint Series, Kyushu University}

The Grobal COE Program

Math-for-Industry Education \& Research Hub

MI

MI2008-1 Takahiro ITO, Shuichi INOKUCHI \& Yoshihiro MIZOGUCHI

Abstract collision systems simulated by cellular automata

MI2008-2 Eiji ONODERA

The intial value problem for a third-order dispersive flow into compact almost

Hermitian manifolds

MI2008-3 Hiroaki KIDO

On isosceles sets in the 4-dimensional Euclidean space

MI2008-4 Hirofumi NOTSU

Numerical computations of cavity flow problems by a pressure stabilized characteristiccurve finite element scheme

MI2008-5 Yoshiyasu OZEKI

Torsion points of abelian varieties with values in nfinite extensions over a padic field

MI2008-6 Yoshiyuki TOMIYAMA

Lifting Galois representations over arbitrary number fields

MI2008-7 Takehiro HIROTSU \& Setsuo TANIGUCHI

The random walk model revisited

MI2008-8 Silvia GANDY, Masaaki KANNO, Hirokazu ANAI \& Kazuhiro YOKOYAMA Optimizing a particular real root of a polynomial by a special cylindrical algebraic decomposition

MI2008-9 Kazufumi KIMOTO, Sho MATSUMOTO \& Masato WAKAYAMA

Alpha-determinant cyclic modules and Jacobi polynomials 
MI2008-10 Sangyeol LEE \& Hiroki MASUDA

Jarque-Bera Normality Test for the Driving Lévy Process of a Discretely Observed Univariate SDE

MI2008-11 Hiroyuki CHIHARA \& Eiji ONODERA

A third order dispersive flow for closed curves into almost Hermitian manifolds

MI2008-12 Takehiko KINOSHITA, Kouji HASHIMOTO and Mitsuhiro T. NAKAO

On the $L^{2}$ a priori error estimates to the finite element solution of elliptic problems with singular adjoint operator

MI2008-13 Jacques FARAUT and Masato WAKAYAMA

Hermitian symmetric spaces of tube type and multivariate Meixner-Pollaczek polynomials

MI2008-14 Takashi NAKAMURA

Riemann zeta-values, Euler polynomials and the best constant of Sobolev inequality

MI2008-15 Takashi NAKAMURA

Some topics related to Hurwitz-Lerch zeta functions

MI2009-1 Yasuhide FUKUMOTO

Global time evolution of viscous vortex rings

MI2009-2 Hidetoshi MATSUI \& Sadanori KONISHI

Regularized functional regression modeling for functional response and predictors

MI2009-3 Hidetoshi MATSUI \& Sadanori KONISHI

Variable selection for functional regression model via the $L_{1}$ regularization

MI2009-4 Shuichi KAWANO \& Sadanori KONISHI

Nonlinear logistic discrimination via regularized Gaussian basis expansions

MI2009-5 Toshiro HIRANOUCHI \& Yuichiro TAGUCHII

Flat modules and Groebner bases over truncated discrete valuation rings 
MI2009-6 Kenji KAJIWARA \& Yasuhiro OHTA

Bilinearization and Casorati determinant solutions to non-autonomous $1+1$ dimensional discrete soliton equations

\section{MI2009-7 Yoshiyuki KAGEI}

Asymptotic behavior of solutions of the compressible Navier-Stokes equation around the plane Couette flow

MI2009-8 Shohei TATEISHI, Hidetoshi MATSUI \& Sadanori KONISHI

Nonlinear regression modeling via the lasso-type regularization

MI2009-9 Takeshi TAKAISHI \& Masato KIMURA

Phase field model for mode III crack growth in two dimensional elasticity

MI2009-10 Shingo SAITO

Generalisation of Mack's formula for claims reserving with arbitrary exponents for the variance assumption

MI2009-11 Kenji KAJIWARA, Masanobu KANEKO, Atsushi NOBE \& Teruhisa TSUDA Ultradiscretization of a solvable two-dimensional chaotic map associated with the Hesse cubic curve

\section{MI2009-12 Tetsu MASUDA}

Hypergeometric T -functions of the q-Painlevé system of type $E_{8}^{(1)}$

MI2009-13 Hidenao IWANE, Hitoshi YANAMI, Hirokazu ANAI \& Kazuhiro YOKOYAMA A Practical Implementation of a Symbolic-Numeric Cylindrical Algebraic Decomposition for Quantifier Elimination

MI2009-14 Yasunori MAEKAWA

On Gaussian decay estimates of solutions to some linear elliptic equations and its applications

MI2009-15 Yuya ISHIHARA \& Yoshiyuki KAGEI

Large time behavior of the semigroup on $L^{p}$ spaces associated with the linearized compressible Navier-Stokes equation in a cylindrical domain 
MI2009-16 Chikashi ARITA, Atsuo KUNIBA, Kazumitsu SAKAI \& Tsuyoshi SAWABE Spectrum in multi-species asymmetric simple exclusion process on a ring

MI2009-17 Masato WAKAYAMA \& Keitaro YAMAMOTO

Non-linear algebraic differential equations satisfied by certain family of elliptic functions

MI2009-18 Me Me NAING \& Yasuhide FUKUMOTO

Local Instability of an Elliptical Flow Subjected to a Coriolis Force 